\title{
Dosimetric evaluation of helical tomotherapy and volumetric-modulated arc therapy for malignant pleural mesothelioma: a planning study for dose escalation
}

\author{
Fu-Li Zhang, Na Lu, Hua-Yong Jiang, Dian-Dian Chen, Ya-Di Wang \\ Department of Radiation Therapy, The Seventh Medical Center of Chinese PLA General Hospital, Beijing, China \\ Contributions: (I) Conception and design: FL Zhang; (II) Administrative support: FL Zhang; (III) Provision of study materials or patients: N Lu; (IV) \\ Collection and assembly of data: HY Jiang; (V) Data analysis and interpretation: DD Chen, YD Wang; (VI) Manuscript writing: All authors; (VII) \\ Final approval of manuscript: All authors. \\ Correspondence to: Fu-Li Zhang, PhD. Department of Radiation Therapy, The Seventh Medical Center of Chinese PLA General Hospital, No. 5 of \\ Nanmencang, Dongcheng District, Beijing 100700, China. Email: fulizhangaz@163.com.
}

\begin{abstract}
Background: To compare the dosimetric differences between helical tomotherapy (HT) and volumetricmodulated arc therapy (VMAT) treatment plans for inoperable malignant pleural mesothelioma (MPM).

Methods: Ten patients with inoperable MPM were retrospectively planned with the HT and VMAT techniques, and the dose-volume histogram (DVH)-based parameters of the planning target volume (PTV) and organs at risk (OARs) were compared.

Results: Compared with the VMAT plans, the target homogeneity index (HI) and conformity index (CI) of the HT plans were significantly better (HI: $1.04 \pm 0.01$ vs. $1.11 \pm 0.03$, CI: $0.80 \pm 0.07$ vs. $0.71 \pm 0.12$, respectively) $(\mathrm{P}<0.001, \mathrm{P}=0.013$, respectively). Regarding the OARs, including the ipsilateral lung, contralateral lung, heart, and spinal cord, the differences among the V30 ( $\mathrm{x}$ : fraction of volume receiving >5, 10, 20, and 30 Gy, respectively) of the ipsilateral lung and V5, V10, and V20 of the contralateral lung were statistically significant $(\mathrm{P}=0.031, \mathrm{P}=0.030, \mathrm{P}=0.021, \mathrm{P}=0.003$, respectively). However, there was no significant differences between HT plans and VMAT plans, regarding the V5, V10 and V20 of the ipsilateral lung, V3 of the contralateral lung, V5 and Dmean of the heart, and Dmax of the cord. The treatment delivery time of the VMAT was significantly shorter than that of the HT (mean delivery time: $3.27 \pm 1.65 v s .11 .11 \pm 3.75 \mathrm{~min}$, respectively) $(\mathrm{P}<0.001)$.

Conclusions: Compared to the VMAT plans, the HT plans not only demonstrated more optimal target coverage and conformity but also considerably reduced the dose-volume parameters of the OARs in both low-dose areas in contralateral lung and high-dose areas in ipsilateral lung and contralateral lung, which is correlated to radiation injury. However, the treatment delivery time of the HT plans was longer.
\end{abstract}

Keywords: Helical tomotherapy (HT); volumetric-modulated arc therapy (VMAT); organs at risk (OARs); dosimetric evaluation; malignant pleural mesothelioma (MPM)

Submitted Jul 01, 2020. Accepted for publication Oct 30, 2020.

doi: $10.21037 /$ tcr-20-2452

View this article at: http://dx.doi.org/10.21037/tcr-20-2452

\section{Introduction}

Although the incidence of malignant pleural mesothelioma (MPM) is low, most lesions are extensive, with obvious symptoms and a poor prognosis. Trimodality therapy including chemotherapy, extrapleural pneumonectomy
(EPP), and hemithorax radiotherapy, has shown promising results in patients with medically operable MPM, with a median survival period of 19-33.5 months in prospective studies (1-5). Findings from a contemporary cohort demonstrated that lung-sparing extended pleurectomy/ 
decortication (P/D) comprised most surgical procedures for MPM compared with EPP (6). However, most cases in China are inoperable at the time of diagnosis; hence, palliative high-dose hemithoracic radiotherapy to the entire ipsilateral pleura with conventional fractionation is used in the management of MPM in the adjuvant, neoadjuvant, and palliative settings (7).

As external beam radiotherapy evolved from threedimensional conformal radiation therapy (3DCRT) to intensity-modulated radiation therapy (IMRT), volumetricmodulated arc therapy (VMAT), and helical tomotherapy (HT), treatment planning and delivery improved greatly. And IMRT/VMAT/HT therapy are being used more commonly now than historically (8). For instance, several studies have shown that conventional static IMRT achieves better dose coverage and improved local control compared with 3DCRT (6-13) for MPM, while other studies have demonstrated that VMAT allows for even better dose conformality in a shorter delivery time compared with static IMRT, sparing potential organs at risk (OARs) during MPM radiotherapy (14-16). Moreover, several studies exploring the feasibility of prescription dose escalation using HT and VMAT have also been reported (17-21). However, little research has been published comparing the differences between HT and VMAT from a dosimetric escalation perspective. Therefore, in this study, we retrospectively analyzed the dosimetric and technical differences between VMAT and HT for MPM in 10 patients previously treated with static IMRT. We quantitatively compared the quality of the treatment plans according to dose uniformity and target volume and dose conformity to the surrounding normal tissue of the ipsilateral lung, contralateral lung, heart, and spinal cord. We present the following article in accordance with the STROBE reporting checklist (available at http://dx.doi.org/10.21037/tcr-20-2452).

\section{Methods}

Patients selection, positioning, and computerized tomography (CT) scanning

Ten patients diagnosed with MPM from September 2006 to May 2013 who were unable to undergo surgery for various reasons were randomly selected as the study subjects. The median age was 48.5 years. All cases were diagnosed as MPM via puncture biopsy combined with immunohistochemistry. Staging was implemented according to the American Joint Committee on Cancer (AJCC) and the Union for International Cancer Control (UICC) seventh edition TNM classification (22), with two patients in stage II, six in stage III, and two in stage IV. For the CT scan, the patients were fixed with a body fixator, placed in a supine position, with both arms raised above their head, and scanned from the level of the larynx to the upper abdomen on a CT simulator (Brilliance Big Bore CT, Philips Medical Systems, Cleveland, USA) with a 5-mm slice thickness and slice separations.

The study was conducted in accordance with the Declaration of Helsinki (as revised in 2013). The study was approved by the ethics board of The Seventh Medical Center of Chinese PLA General Hospital (No. 2020-022) and informed consent was taken from all the patients.

\section{Contouring of target volume and OARs}

The contouring of the target volume and OARs for all patients was performed by a single radiation oncologist with extensive experience in MPM treatment according to the International Commission on Radiation Units and Measurements Report No. 62 (23). The gross target volume (GTV) included the tumors observed in clinical and radiological data, while the planning target volume (PTV) was obtained by expanding the GTV $10 \mathrm{~mm}$ in the direction of the pleura and $5 \mathrm{~mm}$ in the direction of the lungs. The OARs included the ipsilateral lung, contralateral lung, heart, and spinal cord.

\section{Treatment planning}

The HT planning was conducted on the Hi-Art ${ }^{\circledR}$ (version 4.1.2Madison, USA) treatment planning system using field widths of $2.5 \mathrm{~cm}$, a modulation factor of 2.5-3.0, and a pitch of 0.287 or 0.43 . The VMAT planning was completed on the Monaco system (version 5.11, Elekta AB, Stockholm, Sweden) using 6-MV photon beams from an Elekta linac (Synergy, Elekta AB, Stockholm, Sweden) and two partial arcs of $220^{\circ}$. The prescribed dose to the PTV was $60 \mathrm{~Gy}$ in 2-Gy daily fractions. The dose-volume constraints used for the OARs are listed in Table 1.

\section{Plan comparison}

The dosimetric comparison of the plans was performed based on the following parameters extracted from the dosevolume histogram (DVH): the homogeneity index (HI) (24), conformity index (CI) (24), the maximum dose (Dmax), 
the minimum dose (Dmin), and the mean dose (Dmean) of the PTV (Dmax: dose received by $2 \%$ volume of the PTV, Dmin: dose received by $98 \%$ volume of the PTV) (25), V5, V10, V20, and V30 (Vx: fraction of volume receiving $>5,10,20$, and $30 \mathrm{~Gy}$, respectively) of the ipsilateral lung, V3, V5, V10, and V20 of the contralateral lung, V5 and Dmean of the heart, and Dmax of the spinal cord. Figure 1 displays the isodose distributions of the HT and VMAT radiotherapy plans for a typical patient.

\section{Statistical analysis}

The Wilcoxon signed rank test was used to compare the results of the two types of plans using the SPSS 20 software (SPSS Inc., Chicago, USA). The quantitative data were

Table 1 Dose-volume constraint for OARs

\begin{tabular}{lcc}
\hline OARs & Dose (Gy) & Volume $(\%)$ \\
\hline Ipsilateral lung & 10 & $<70$ \\
& 20 & $<50$ \\
Contralateral lung & 10 & $<40$ \\
Heart & 5 & $<60$ \\
Cord & 45 & $<1$ \\
\hline
\end{tabular}

OARs, organs at risk. expressed in the form of the mean \pm standard deviation $(\bar{x} \pm s)$. A value of $\mathrm{P}<0.05$ was considered statistically significant.

\section{Results}

\section{Comparison of the HI, CI, Dmax, Dmin, and Dmean for} the HT and VMAT

The DVH plots of the PTV of the HT and VMAT plans of a typical patient were exported into the Computational Environment for Radiological Research software and are illustrated in Figure 2. The HI, CI, Dmax, Dmin, and Dmean of the plans are presented for comparison in Table 2. Significant differences were found in the Dmax, Dmin, Dmean, HI, and CI values between the HT and VMAT plans $(\mathrm{P}=0.002, \mathrm{P}=0.010, \mathrm{P}=0.002, \mathrm{P}=0.002$, $\mathrm{P}=0.004$, respectively). Overall, the HT plans provided significantly better uniformity and conformity than the VMAT plans.

The DVH plots of the OARs of the HT and VMAT plans of a typical patient are illustrated in Figure 3. The doses and irradiated volume parameters of the ipsilateral lung, contralateral lung, heart, and spinal cord are listed in Table 3. The V30 of the ipsilateral lung and V3, V10, and V20 of the contralateral lung all demonstrated significant differences $(\mathrm{P}=0.037, \mathrm{P}=0.043, \mathrm{P}=0.039, \mathrm{P}=0.004$,
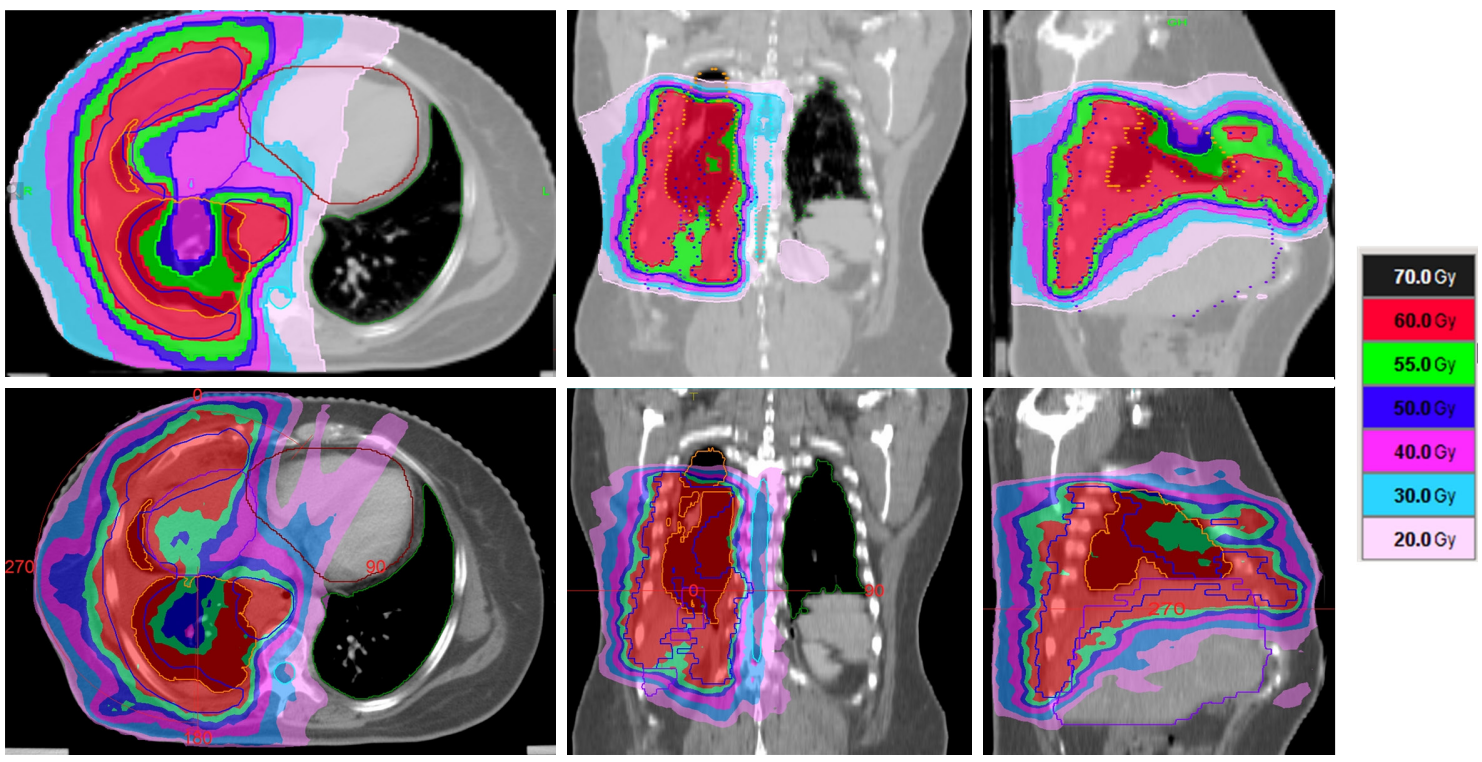

$20.0 \mathrm{~Gy}$

Figure 1 The isodose distributions of HT and VMAT radiotherapy plans for a typical patient in transverse, coronal, sagittal view (upper: HT, lower: VMAT). HT, helical tomotherapy; VMAT, volumetric-modulated arc therapy. 
respectively). However, there was no significant differences between HT plans and VMAT plans, regarding the V5, V10 and V20 of the ipsilateral lung, V5 of the contralateral lung, V5 and Dmean of the heart, and Dmax of the cord $(\mathrm{P}=0.098$, $\mathrm{P}=0.846, \mathrm{P}=0.084, \mathrm{P}=0.064, \mathrm{P}=0.313, \mathrm{P}=0.131, \mathrm{P}=0.105$, respectively).

\section{Treatment delivery efficiency}

The efficiency of the two plan types was evaluated by the

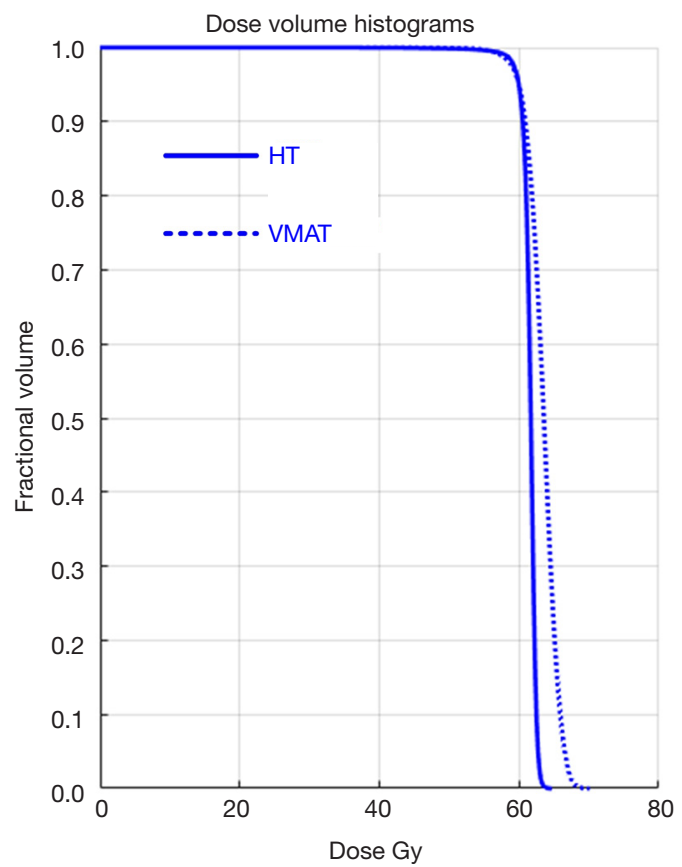

Figure 2 DVH plots of PTV of the HT and VMAT plans of a typical patient. DVH, dose-volume histogram; PTV, planning target volume; HT, helical tomotherapy; VMAT, volumetricmodulated arc therapy. number of monitor units (MUs) and the delivery time (Table 4). The number of MUs in the HT plan was much higher than that in the VMAT plan, and the former's treatment delivery time was $239.8 \%$ longer than that of the latter. The treatment delivery time of the VMAT was significantly shorter than that of the HT (mean delivery time: $3.27 \pm 1.65$ vs. $11.11 \pm 3.75 \mathrm{~min}$, respectively) $(\mathrm{P}=0.002)$.

\section{Discussion}

A novel IMRT system that integrates spiral CT technology with linear accelerator technology, HT implements delivery in a $360^{\circ}$ range at all angles. In the irradiation process, the gantry rotation, table motion, accelerator pulse, and opening and closing of the binary multileaf collimator (MLC) are synchronized (26). Meanwhile, VMAT is based on the development of fixed-field IMRT and image-guided radiation therapy technology and differs from conventional static IMRT in that it can simultaneously change the rotation speed of the gantry, the MLC position, and the dose rate to achieve a highly conformal dose distribution.

In previous research, Helou et al. used HT to treat 29 patients with MPM with a median prescription dose of 50 Gy and 2 Gy per fraction. The V2, V5, V10, V13, and V20 of the lungs in the treatment plan were $100 \%$, $98 \%, 52 \%, 36 \%, 19 \%$, and $5 \%$, respectively. They found that when V10 of the contralateral lung was greater than $50 \%$, the risk of radiation pneumonia tended to increase (27). Minatel et al. treated 28 MPM patients with HT, delivering a prescribed dose of 50 Gy to the PTV. Any fluorodeoxyglucose-avid areas or regions of particular concern for residual disease were given a simultaneous boost of radiotherapy up to $60 \mathrm{~Gy}$. Three patients had grade 2 radiation pneumonitis, and two had grade 3 radiation pneumonitis. The $\mathrm{V} 5$ of the contralateral lung was $32 \%$-far higher than those who did not develop radiation

Table 2 Comparison of HI, CI, Dmax, Dmin, Dmean for HT and VMAT $\left(\bar{x}_{ \pm \mathrm{S}}\right)$

\begin{tabular}{lcccc}
\hline Parameters & HT & VMAT & Z value & P value \\
\hline Dmax & $63.15 \pm 0.99$ & $67.22 \pm 2.21$ & -2.803 & 0.002 \\
Dmin & $59.26 \pm 0.46$ & $58.61 \pm 0.72$ & -2.497 & 0.010 \\
Dmean & $61.82 \pm 0.68$ & $63.27 \pm 1.04$ & -2.803 & 0.002 \\
$\mathrm{HI}$ & $1.04 \pm 0.01$ & $1.11 \pm 0.03$ & -2.814 & 0.002 \\
$\mathrm{Cl}$ & $0.80 \pm 0.07$ & $0.71 \pm 0.12$ & -2.703 & 0.004 \\
\hline
\end{tabular}

$\mathrm{HI}$, homogeneity index; $\mathrm{Cl}$, conformity index; Dmax, dimension maximum; Dmin, dimension minimum; Dmean, dimension mean; HT, helical tomotherapy; VMAT, volumetric-modulated arc therapy. 

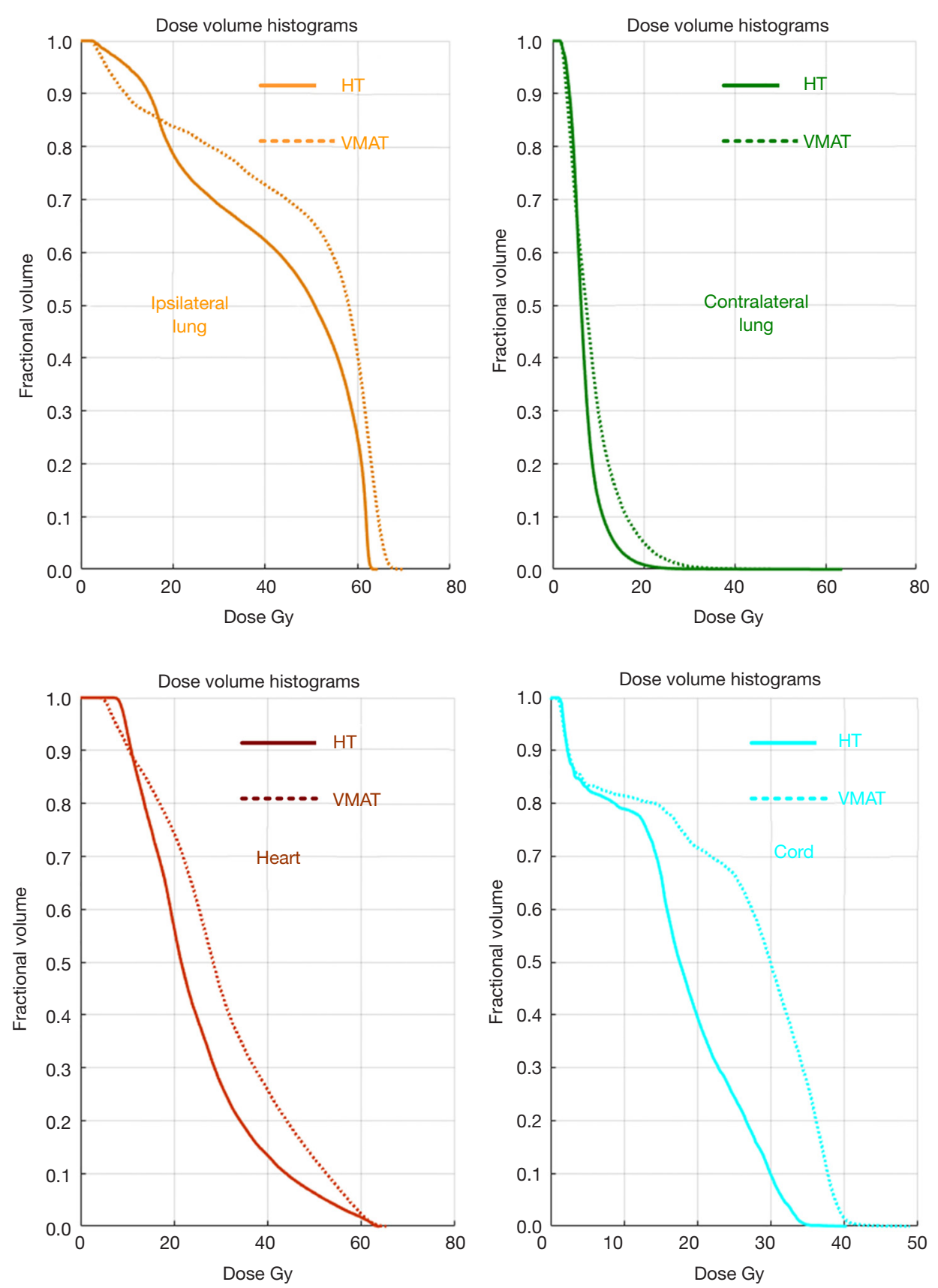

Figure 3 DVH plots of OARs of the HT and VMAT plans of a typical patient. DVH, dose-volume histogram; OARs, organs at risk; HT, helical tomotherapy; VMAT, volumetric-modulated arc therapy.

pneumonitis (V5 =17\%) (28). Harrabi et al. used HT in 10 patients with MPM who had undergone pleurectomy/ decortication $(\mathrm{P} / \mathrm{D})$, with a median prescription dose of 52.2 Gy. The minimum lethal dose (MLD) and V20 of the ipsilateral lung were 32.8 Gy and $71.7 \%$, respectively. No treatment-related toxicity exceeding grade III according to the common toxicity criteria was observed (29). Hong et al. treated 11 patients who had received neoadjuvant chemotherapy after surgery with $\mathrm{HT}$ at a prescription dose of $25 \mathrm{~Gy}$ in five fractions to the entire ipsilateral hemithorax. 
Table 3 Comparison of dosimetric parameters of OARs for HT and VMAT $\left(\bar{x}_{ \pm \mathrm{s}}\right)$

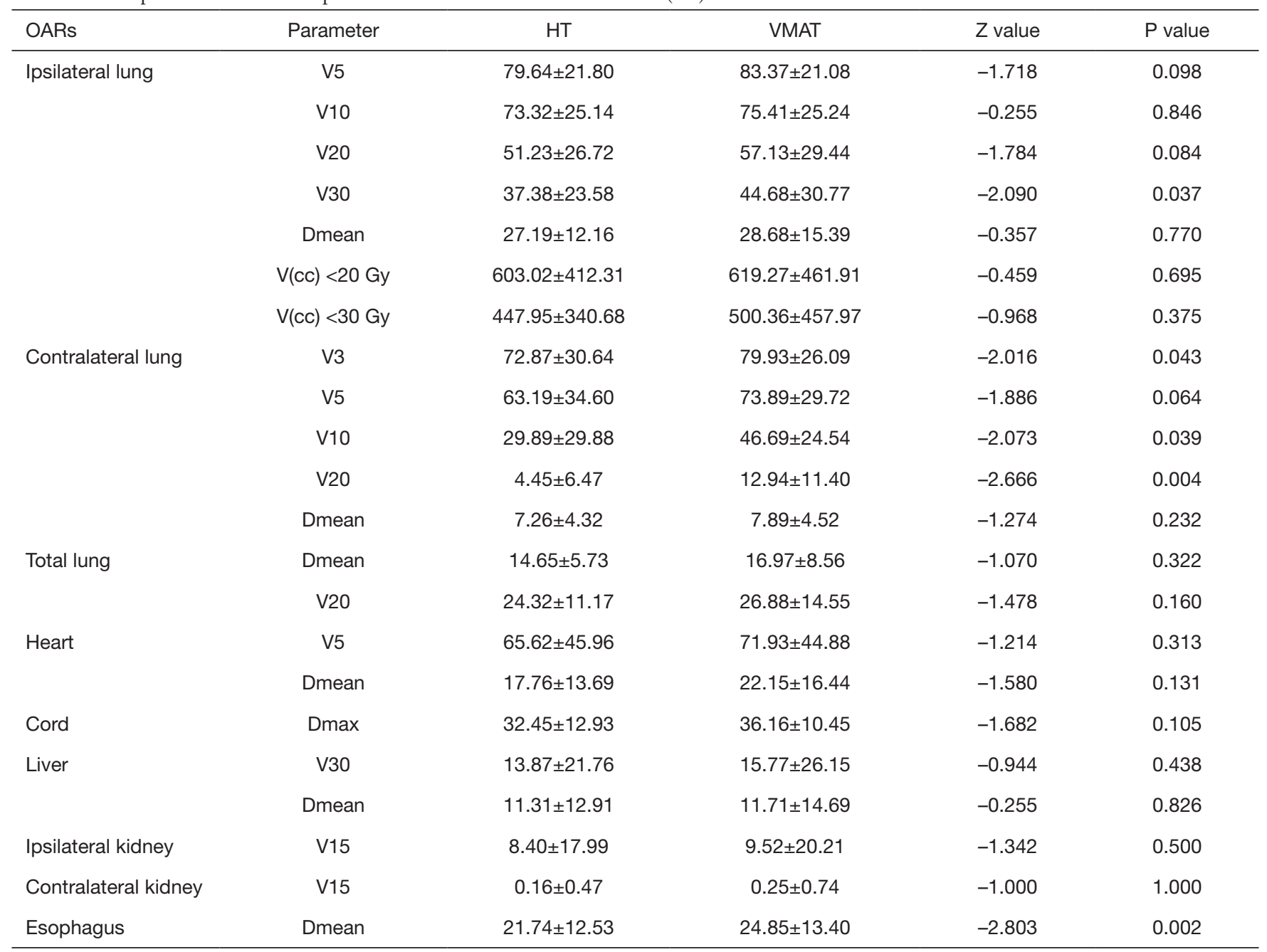

OARs, organs at risk; HT, helical tomotherapy; VMAT, volumetric-modulated arc therapy.

Table 4 Comparison of MU and treatment delivery time $\left(\bar{x}_{ \pm s}\right)$

\begin{tabular}{lcccc}
\hline Parameter & HT & VMAT & Z value & P value \\
\hline MUs & $9,776.8 \pm 3,301.6$ & $907.6 \pm 378.9$ & -2.803 & 0.002 \\
Treatment time $(\min )$ & $11.11 \pm 3.75$ & $3.27 \pm 1.65$ & -2.803 & 0.002 \\
\hline
\end{tabular}

MUs, monitor units; HT, helical tomotherapy; VMAT, volumetric-modulated arc therapy.

The MLD and V7 of the contralateral lung were lower than 3.5 Gy and 20\%, respectively, and only one patient developed acute grade 3 radiation pneumonitis (30). Leitzen et al. performed an HT planning study on the datasets of 13 patients who received radiotherapy after pleurectomy, with an applied dose to the PTV of 50.4 Gy and single doses of 1.8 Gy per fraction. For the PTV (left-sided/right-sided), the Dmin was 49.37/49.71 Gy (98.0\%/98.6\%), and the Dmax was 54.19/54.61 Gy (107.5\%/108.3\%). The beam-on time was kept below $15 \mathrm{~min}$. The MLD of the contralateral lung was below $4 \mathrm{~Gy}$, and the Dmean of the heart was 22.23 Gy (31). Kimura et al. treated a total of 15 patients after EPP with VMAT. The dose prescription was designed to cover $95 \%$ of the PTV with 54 Gy in 30 fractions. The 
MLD, V5 and V20 of the contralateral lung were 6.4 Gy (range, 5.2-8.2 Gy), 45.9\% (range, 29.3-57.7\%) and 2.1\% (range, $0.1-6.6 \%$ ), respectively. Grade 3 pneumonitis after the treatment was observed in three patients (20.0\%) (14). A recent study compared HT and VMAT in terms of dosimetric parameters in positron emission tomography-CT-based radiation therapy in unresectable MPM. The PTV1 and PTV2 prescription doses were 45.0 and 54 Gy, respectively, in 1.8 Gy per fraction. For the HT plan, the V5, V10, and V20 of the ipsilateral lung were $99.7 \%, 95.1 \%$, and $80.2 \%$, respectively, while the $\mathrm{V} 5, \mathrm{~V} 10$, and V20 of the contralateral lung reached $30.6 \%$, $29.6 \%$, and $0.5 \%$, respectively. The Dmean of the heart was 25.8 Gy, and the Dmax of the cord was 33.2 Gy. For the VMAT plan, the V5, V10, and V20 of the ipsilateral lung were $100 \%, 100 \%$, and $92 \%$, respectively, while the V5, V10, and V20 were $67.8 \%, 51.4 \%$, and $3.5 \%$, respectively. The Dmean of the heart was $33.3 \mathrm{~Gy}$, and the Dmax of the cord was 39.3 Gy (31). Both the Dmax and Dmean of the PTV1 and PTV2 favored the HT plan over the VMAT plan. Additionally, the HT also provided more homogeneous dose distribution and numerically lower doses received by most OARs. The primary disadvantage of the HT technique was the requirement for longer treatment times (7.4 vs. 2.5 minutes/fraction).

In this retrospective dosimetric study, to explore the feasibility of escalating the prescription radiotherapy dose for patients suffering inoperable MPM, the prescribed PTV dose was determined to be $60 \mathrm{~Gy}$ in all cases, which has not yet been applied in clinical work. When $95 \%$ of the PTV received the prescribed dose of $60 \mathrm{~Gy}$, the dose-volume parameters of some patients' lungs significantly exceeded the tolerated dose limits of today's clinic routine, so some plans could not be used for clinical implementation. This is a limitation of the study.

Herein, the target area conformity and dose uniformity of the HT treatment plan were better than those of the VMAT plan, and the dose-volume parameters of the OARs of the former were slightly lower than those of the latter. Regarding the HT plans, the V5, V10, and V20 of the ipsilateral lung, Dmean of the heart, and Dmax of the cord were $79.6 \%, 73.3 \%, 51.23 \%, 17.8 \mathrm{~Gy}$, and $32.5 \mathrm{~Gy}$, respectively, all of which were lower than the corresponding values in Pehlivan's study (32). The V5 and V20 of the contralateral lung were $63.2 \%$ and $4.5 \%$, respectively, higher than the corresponding values in Pehlivan's study, while the V10 was similar in both studies. Regarding the VMAT plans, the V5, V10, and V20 of the ipsilateral lung, Dmean of the heart, and Dmax of the cord were 83.4\%, 75.4\%, 57.1\%, 22.2 Gy, and 36.2 Gy, respectively, all of which were lower than the corresponding values in Pehlivan's study. The V5 and V20 of the contralateral lung were $73.9 \%$ and $12.9 \%$, respectively, higher than the corresponding values in Pehlivan's study, while the V10 was again similar in both. However, HT technology still has deficiencies in clinical treatment operation, as the segment number in an HT plan significantly lengthens the treatment time, making it difficult to fully ensure the patient's comfort and setup repeatability throughout.

\section{Conclusions}

This dosimetric study demonstrated the possibility of precise hemithoracic irradiation of medically/technically inoperable MPM patients with either HT or VMAT. These novel radiotherapy techniques have great potential to transform technological advantages into therapeutic benefits in future clinical work.

\section{Acknowledgments}

We would like to acknowledge the hard and dedicated work of all the staff that implemented the intervention and evaluation components of the study.

Funding: The study was supported by the Special Project of Military Medical Metrology: research on the implementation plan of clinical application quality control of tomotherapy (No. 2011-j12-005) and Beijing Municipal Science and Technology Commission (No. Z181100001718011).

\section{Footnote}

Reporting Checklist: The authors have completed the STROBE reporting checklist. Available at http://dx.doi. org/10.21037/tcr-20-2452

Data Sharing Statement: Available at http://dx.doi. org/10.21037/tcr-20-2452

Conflicts of Interest: All authors have completed the ICMJE uniform disclosure form (available at http://dx.doi. org/10.21037/tcr-20-2452). The authors have no conflicts of interest to declare.

Ethical Statement: The authors are accountable for all 
aspects of the work in ensuring that questions related to the accuracy or integrity of any part of the work are appropriately investigated and resolved. The study was conducted in accordance with the Declaration of Helsinki (as revised in 2013). The study was approved by the ethics board of The Seventh Medical Center of Chinese PLA General Hospital (No. 2020-022) and informed consent was taken from all the patients.

Open Access Statement: This is an Open Access article distributed in accordance with the Creative Commons Attribution-NonCommercial-NoDerivs 4.0 International License (CC BY-NC-ND 4.0), which permits the noncommercial replication and distribution of the article with the strict proviso that no changes or edits are made and the original work is properly cited (including links to both the formal publication through the relevant DOI and the license). See: https://creativecommons.org/licenses/by-nc-nd/4.0/.

\section{References}

1. Sugarbaker DJ, Flores RM, Jaklitsch MT, et al. Resection margins, extrapleural nodal status, and cell type determine postoperative long-term survival in trimodality therapy of malignant pleural mesothelioma: results in 183 patients. J Thorac Cardiovasc Surg 1999;117:54-63; discussion 63-5.

2. Batirel HF, Metintas M, Caglar HB, et al. Trimodality treatment of malignant pleural mesothelioma. J Thorac Oncol 2008;3:499-504.

3. Weder W, Stahel RA, Bernhard J, et al; Swiss Group for Clinical Cancer Research. Multicenter trial of neo-adjuvant chemotherapy followed by extrapleural pneumonectomy in malignant pleural mesothelioma. Ann Oncol 2007;18:1196-202.

4. Flores RM, Krug LM, Rosenzweig KE, et al. Induction chemotherapy, extrapleural pneumonectomy, and postoperative high-dose radiotherapy for locally advanced malignant pleural mesothelioma: a phase II trial. J Thorac Oncol 2006; 1:289-95.

5. Rea F, Marulli G, Bortolotti L, et al. Induction chemotherapy, extrapleural pneumonectomy (EPP) and adjuvant hemi-thoracic radiation in malignant pleural mesothelioma (MPM): feasibility and results. Lung Cancer 2007;57:89-95.

6. Thieke C, Nicolay NH, Sterzing F, et al. Long-term results in malignant pleural mesothelioma treated with neoadjuvant chemotherapy, extrapleural pneumonectomy and intensity-modulated radiotherapy. Radiat Oncol
2015;10:267.

7. Rice DC, Smythe WR, Liao Z, et al. Dose-dependent pulmonary toxicity after postoperative intensity-modulated radiotherapy for malignant pleural mesothelioma. Int J Radiat Oncol Biol Phys 2007;69:350-7.

8. Shaaban SG, Verma V, Choi JI, et al. Utilization of intensity-modulated radiation therapy for malignant pleural mesothelioma in the United States. Clin Lung Cancer.2018;19:e685-92.

9. Tonoli S, Vitali P, Scotti V, et al. Adjuvant radiotherapy after extrapleural pneumonectomy for mesothelioma. Prospective analysis of a multi-institutional series. Radiother Oncol 2011;101:311-5.

10. Ahamad A, Stevens CW, Smythe WR, et al. Intensitymodulated radiation therapy: a novel approach to the management of malignant pleural mesothelioma. Int J Radiat Oncol Biol Phys 2003;5 5:768-75.

11. Buduhan G, Menon S, Aye R, et al. Trimodality therapy for malignant pleural mesothelioma. Ann Thorac Surg 2009;88:870-5; discussion 876.

12. Du KL, Both S, Friedberg JS, et al. Extrapleural pneumonectomy, photodynamic therapy and intensity modulated radiation therapy for the treatment of malignant pleural mesothelioma. Cancer Biol Ther 2010;10:425-9.

13. Krayenbuehl J, Dimmerling P, Ciernik IF, et al. Clinical outcome of postoperative highly conformal versus $3 \mathrm{D}$ conformal radiotherapy in patients with malignant pleural mesothelioma. Radiat Oncol 2014;9:32.

14. Kimura T, Doi Y, Nakashima T, et al. Clinical experience of volumetric modulated arc therapy for malignant pleural mesothelioma after extrapleural pneumonectomy. J Radiat Res 2015;56:315-24.

15. Scorsetti M, Bignardi M, Clivio A, et al. Volumetric modulation arc radiotherapy compared with static gantry intensity-modulated radiotherapy for malignant pleural mesothelioma tumor: a feasibility study. Int J Radiat Oncol Biol Phys 2010;77:942-9.

16. Runxiao L, Yankun C, Lan W. A pilot study of volumetricmodulated arc therapy for malignant pleural mesothelioma. J Appl Clin Med Phys 2016;17:139-44.

17. Ebara T, Kawamura H, Kaminuma T, et al. Hemithoracic intensity-modulated radiotherapy using helical tomotherapy for patients after extrapleural pneumonectomy for malignant pleural mesothelioma. J Radiat Res 2012;53:288-94.

18. Renaud J, Yartsev S, Dar AR, et al. Adaptive radiation therapy for localized mesothelioma with mediastinal 
metastasis using helical tomotherapy. Med Dosim 2009;34:233-42.

19. Maggio A, Cutaia C, Di Dia A, et al. Tomotherapy PET-guided dose escalation: a dosimetric feasibility study for patients with malignant pleural mesothelioma. Strahlenther Onkol 2016;192:102-8.

20. Fodor A, Broggi S, Incerti E, et al. Moderately hypofractionated helical IMRT, FDG-PET/CT-guided, for progressive malignant pleural mesothelioma in patients with intact lungs. Clin Lung Cancer 2019;20:e29-38.

21. Botticella A, Defraene G, Nackaerts K, et al. Does selective pleural irradiation of malignant pleural mesothelioma allow radiation dose escalation?: a planning study. Strahlenther Onkol 2017;193:285-94.

22. Kindler HL. Robust data: the essential foundation of a revised staging system for pleural mesothelioma. J Thorac Oncol 2012;7:1623-4.

23. Chavaudra J, Bridier A. Definition of volumes in external radiotherapy: ICRU reports 50 and 62. Cancer Radiother 2001;5:472-8.

24. Weiss E, Siebers JV, Keall PJ. An analysis of 6-MV versus 18-MV photon energy plans for intensity-modulated radiation therapy (IMRT) of lung cancer. Radiother Oncol 2007;82:55-62.

25. Hodapp N. The ICRU Report 83: prescribing, recording and reporting photon-beam intensity-modulated radiation therapy (IMRT). Strahlenther Onkol 2012;188:97-9.

26. Bortfeld T, Webb S. Single-arc IMRT? Phys Med Biol

Cite this article as: Zhang FL, Lu N, Jiang HY, Chen DD, Wang YD. Dosimetric evaluation of helical tomotherapy and volumetric-modulated arc therapy for malignant pleural mesothelioma: a planning study for dose escalation. Transl Cancer Res 2021;10(2):914-922. doi: 10.21037/tcr-20-2452
2009;54:N9-20.

27. Helou J, Clement-Colmou K, Sylvestre A, et al. Helical tomotherapy in the treatment of malignant pleural mesothelioma: the impact of low doses on pulmonary and oesophageal toxicity. Cancer Radiother 2013;17:755-62.

28. Minatel E, Trovo M, Polesel J, et al. Tomotherapy after pleurectomy/decortication or biopsy for malignant pleural mesothelioma allows the delivery of high dose of radiation in patients with intact lung. J Thorac Oncol 2012;7:1862-6.

29. Harrabi SB, Koerber SA, Adeberg S, et al. Malignant pleural mesothelioma--pleural cavity irradiation after decortication with helical tomotherapy. Rep Pract Oncol Radiother 2017;22:402-7.

30. Hong JH, Lee HC, Choi KH, et al. Preliminary results of entire pleural intensity-modulated radiotherapy in a neoadjuvant setting for resectable malignant mesothelioma. Radiat Oncol J 2019;37:101-9.

31. Leitzen C, Wilhelm-Buchstab T, Stumpf S, et al. Tomotherapy in malignant mesothelioma: a planning study to establish dose constraints. Strahlenther Onkol 2019;195:668-76.

32. Pehlivan B, Sengul K, Yesil A, et al. Dosimetric comparison of lung-sparing radiation therapy between volumetric arc therapy and helical tomotherapy for unresectable malignant pleural mesothelioma. Biomed Res Int 2019;2019:4568958. 